technical support, customer service, and so on. For these people, computerized systems represent powerful tools for assisting them in the performance of their work. The problems derive from the increasing ability of these systems to take over much of the work now performed by manufacturing, clerical, and service workers.
Solutions will not be simple. Elements leading towards acceptable solutions must include human considerations in the direction and pace of change; shortening individual work time to spread available jobs around more widely; and creation of new products and services both to create additional jobs and to meet social needs.

\title{
An Intelligent Decision Support System for Editors
}

\author{
Manfred Kochen
}

Computer and Information Systems Department, Graduate School of Business Administration, University of Michigan, Ann Arbor, MI 48109, USA

Journal publication is still the primary vehicle by which scholars and scientists communicate and build a cumulative record. But it is only a question of time before economic pressures of rising publication costs and the opportunities made possible by information technologies for new, more costeffective publication options will effect major changes in the very concept of 'journal publication'. To begin with, the idea of 'publication' may be transformed into a notion that admits of 'degree of public domain'. The concepts of 'journal' which literally means 'daily', or more generally 'periodical', may not be necessary altogether or of secondary im-

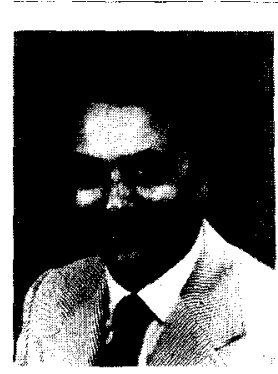

Manfred Kochen was educated at MIT with a B.S. in science and at $\mathrm{Col}$ umbia University in New York, where he received an MA in 1951 in Applied Mathematics. Since 1972 he has been Professor of Information Science, with a joint appointment as $\mathrm{Ad}$ junct Professor of Computers and Information Systems at the Graduate School of Business Administration and as Research Scientist at the Mental Health Research Institute. His major research interests are in problems of representation of knowledge that could help people cope with a greater variety of tasks in more effective ways as well as in the organization of knowledge. portance. What is primarily at issue is the distribution of knowledge with regard to accurate targeting, screening and organization according to relevance, importance, quality, novelty, and comprehensibility.

In what follows, we sketch a series of possible changes from the present system of scientific periodicals to a new kind of communication system with structures and functions broadly analogous to, and beyond, those of a higher central nervous system or brain, possibly the next evolutionary stage.

\section{An Expert System to Advise Editors in Referee-Selection}

This is the first system in the series. It only goes a small step beyond present practice. We take the current system of periodicals to be characterized by the following variables:

$T$ : the time (days) between issues: e.g. 90 for a quarterly

$L$ : the number of articles per issue: typically, 5-10

$q^{*}$ : the minimum quality for an article to be published based on consensus among referees on some rating scale $c$ : circulation or number of people exposed to an issue

$i$ : the impact factor, multiplied by the number of people impacted (Garfield, 1972).

One of the editor's key tasks is to decide about acceptance or rejection of manuscripts. This is generally with the advice of a set of referees. A key goal is to minimize the probabilities of erroneously rejecting a high-quality paper (which may subsequently appear in a competitive periodical) and of erroneously accepting low-quality papers. In a previous paper (Kochen, 1983) we analyzed a proposec means of decreasing these two probabilities to achieve a level of acceptable risk: The idea is to view referees as a sample of the most competent potential readership and to increase the sample size for each manuscript. The essential idea was to provide the editor with an expert system that advises him in the selection of such a sample of referees, and that supports him in the administration of getting usable reports from them expeditiously. Traditionally, this expert system consists of the editor's memory augmented by the publication's Editorial Board. The knowledge base consists of activereferee profiles, constantly-updated profiles of potential referees, and if-then rules that hypothesize how a given kind of referee would react in general to a given kind of manuscript.

A practical implementation of such a system might work as in the following algorithm:

1. Ask the authors of a manuscript to name as many peers as they can think of, whose judgment they would respect. Ask the persons so named to provide their own lists of experts on the topic of the manuscript. Continue this snowball sampling procedure until there is evidence of closure. If data about who nominated or cited whom in what specialty were pre-recorded, this procedure could be simulated by computer. The result is a sizable population of potential readers to sample from.

2. To sample such a population with a given manuscript, the editor must know enough about the manuscript or its author to associate or group them with others that can serve as an entry point, in case the author is not in the target population. 
3. To build, update and use such a database, the editor also uses a knowledge base containing statements about the topics of various manuscripts that help him relate the topic of the manuscript to the topics on which the potential referees are experts and to make inferences about the probable quality of various referees' judgments.

4. A random sample of $n$ is chosen from a set of equally qualified candidatereferees. In doing this, a database about the referees, including their promptness, interests, case loads, etc. is used.

5. The process of search, selection, contacting of candidates, negotiating with them for a prompt, thorough reply, thanking them, etc. is handled by applications programming using a (computerized) database management system.

6. Using tests of the kind developed by Tversky, Kahnemann, etc. the editor determines possible biases in referees' judgments, compensates for them, and combines diverse judges' outputs into a composite. That, together with estimates of the probability that a decision to accept when the manuscript is of low quality (plus estimated cost of consequences) is used by the editor to support his decision.

It is clear that if sample size $n$ is sufficiently large, quality of publications should increase.

\section{An On-line Community of Authors and Peers}

It is only a small conceptual step from the above system to replace much of the written media in communication by electronic means, including the physical manuscript. Assume that an entire scientific community were on-line, with each member having easy access to his own workstation wherever and whenever he needed it and able to easily access a computer conferencing system at little or no cost, so that he does it naturally and regularly. An author, having composed his manuscript with the help of a wordprocessing system on his workstation, sends it to the editor as he would transmit electronic mail. The latter scans it on his monitor. Perhaps he also uses a content analysis program to supplement the key words supplied by the author. These are entered into the knowledge base to generate a set of topics on which peers might be experts. The authors of references cited in the paper are entered into a citation database to generate a list of candidate referees. Their expertise/interest-profiles are checked against the generated topic-keywords. The latter are used to search an index, and that may add to the pool of candidate referees. That pool is expanded by use of the database that lists for some of its members whom they nominate or cite as experts. The final pool members are screened for availability, interest, etc. based on stored data. If that data is outdated or absent or doubtful, the candidates are queried via electronic mail. A random sample is chosen from the finalized pool, the size fixed so as to produce the desired estimated probability of error.

The referees are contacted on-line, and if all agree, a computer conference is started. The editor may invite the author to join at an appropirate time. No one needs to reveal his identity. The editor is conference organizer or he may ask one of the referees to serve as such. His task is to keep the conference focussed and on a time schedule, to keep participants from being overloaded and to render a consensual judgment. If the editorial decision is to revise, there can be sufficient on-line dialogue with the authors, including online presentation of the revisions, until an acceptance/rejection decision is reached.

It is clear that decision quality and quality of accepted manuscripts should improve. The time to reach a final decision should be reduced. Though perhaps the referees may have to spend more time, it may be far more rewarding. This last consideration reflects the major problem of exponential publication growth: overloading of referee candidates, restriction of their field in which they exercise judgement as a coping mechanism, and finer-grained compartmentalization of knowledge. (A subsequent coping mechanism in the feedback loops is the effort to generate dialogue and scientific inquiry on interdisciplinary pathways, linking compartments. But in an institutional universe which generates incentives and rewards based on specialization, penalizing all but extraordinary cases of interdisciplinary work, such efforts are usually quixotic and frequently commercially and professionally penalized.) Indeed, if the low costs of telecommunication and data-handling technologies make the conferencing mechanism sufficiently attractive to publishers and editorial boards, one can envision an operating environment in which requests to participate in on-line conferences become as numerous and irritating as refereeing requests had been during the era of print media. The design and availability of expert systems may minimize the transaction costs of quality control in scientific dialogues, but they will clarify and heighten awareness of the currently irreducible costs of making judgment calls through the application of human expertise.

\section{Non-periodical Distribution}

In the above we have still assumed batch processing toward a final printed output at periodic intervals. If all the readers are on-line, that may not be necessary. Newsworthy compacts could be transmitted electronically as soon as they are ready to enter public domain, as in this compact journal. Archival material is stored via mass storage for retrieval on request. Let us assume that manuscripts are still published in batches or issues. But now, we assume a pipeline of manuscripts that arrive at varying times and are decided upon at a random time later. The accepted manuscripts vary in quality.

The editor/publisher can decide to publish an issue only whenever he has a fixed number of manuscripts in the pipeline, each of which exceeds a certain quality. (That quality threshold may differ from the one used for the publishability decision, and may vary from issue to issue or publication to publication.) Consequently, the time between issues will be a random variable.

Alternatively, the editor could let $L$, the number of manuscripts per issue, vary, and publish an issue every $T$ days with each article in the issue of a quality exceeding $q^{*}$. Here $L$ is a random variable with some issues having 1 article, others 10 or more.

A third alternative is to let both $L$ and $T$ vary, and aggregate articles into an issue only if they fit together topically, logically, qualitatively, or according to some other organizing principle and if 
their quality is high enough. This would very probably increase $i$, the impact, and consequently increase circulation.

Questions do arise concerning the definitions applying to 'circulation' and its relationship to 'impact' as publishing shifts to the environment so described. A business manager must define impact in terms of the profitability realized through the dissemination of new knowledge to a community, which he or she profiles as a market. However, a scientist or research administrator would prefer definitions of 'impact' reflecting transactions of the most relevant possible material for his projects and/or staff. Ironically, a central periodical library facility may be laboring under crippling budget constraints from the point of view of its managers and advisors. Yet an intelligent surveying of the user community might well demonstrate that each individual buys - not to mention having access to - more periodical literature than time permits even to scan optimally. Hence the linkage between 'most relevant' and an operational definition of 'impact' in a scientific environment. As demand publishing, which I describe next, becomes an online reality, control over dissemination to the business manager's 'market' becomes a nightmare and his measure of 'impact' will bear less and less upon the scientific impact. Instead of a single subscriber, or a handful of physically local users of a central periodical facility, we envision delivery of electronic text to some point in a local area net from which dozens, if not hundreds, of members of the market can tap it. It is in the administration of cost and copyright considerations that assume top priority in such a system that 'publishers', or their future role equivalents, will find some of their most urgent managerial challenges.

\section{Demand Publishing}

As conceived here, this differs from an electronic version of the old idea of publishing separates. That idea had been tried and was found to be uneconomical. Readers would request and pay for reprints of selected papers on the basis of a periodically issued table of contents (somewhat like Current Contents). Inventory and account management was, however, too costly to justify the system at prices readers would pay. Presumably computerized management and the use of high-speed electronically-driven printers would now change this cost picture. It would be even more attractive if the requested article could be transmitted over a high-bandwidth channel (optical cables or satellite) from a video disk with archival storage.

But this still assumes batch publishing. Why not broadcast news of an item the moment the publication decision is made, with immediate availability of part or all of the text as soon as it is requested?

Better yet, why not, somewhat as in 'Automatic Science Citation Alerts', maintain interest profiles of potential readers and, instead of broadcasting news and hoping they are paying attention, alert them individually to the news? Then transmit to them the part or all of the article, should they request it. Finally, elicit some response from the reader in order to increase the sensitivity and accuracy of their profile so that future targeting can be more accurate, as in DICO (Flood \& Kochen, 1965) and SASIDS (Flood, 1965). The key problem in such sociotechnological systems is to ensure that the number of productions of important news and their consumption remains balanced. We want neither an oversupply relative to consumption nor an excess of demand over supply, which caused the SASIDS experiment to fail. Each member in the net should be both a producer and a consumer of news so that it becomes an exchange net.

\section{Degrees of Publication}

The instant an author submits a manuscript to the editor of a journal, he agrees to subject it to public scrutiny and gives up rights to his exclusively private use of its contents. But by revealing it only to $n$ referees, the editor restricts the public to whom the manuscript is exposed, and the referees are generally assumed not to divulge all aspects of the manuscript widely or to use it for their own private gain. In the system described in the previous section, the manuscript reaches a self-selected readership which happens also in a conventional publication - and that is a restricted public, too.
Moreover, the author, editor, the system or the reader may control the portion of a manuscript that is revealed to him at any time. If the manuscript is written in the pyramidal style favored by journalists, then the headline is revealed first. Should the reader want more, the lead paragraph comes next, followed by a hierarchic unfolding into the minute details. In this process, the author may block certain segments from the view of certain classes of readers.

Some of the filters or masks may be specified, designed or adjusted by each reader, individually. Other filters are controlled by the author and/or the editor. Referee and reader comments may or may not be available as part of a manuscript, subject to various negotiations and agreements. These filters are in the form of software that is adjusted by each indivudual at his workstation, much as there can be multiple views of a database in current database management systems.

\section{The Nerves of a Scien- tific Community}

If a potential reader has not entered or updated his interest profile he cannot be an eligible recipient of news about someone else's contribution. If a contributor feels that certain others 'ought to' be interested in his contribution, even if they were not aware of this, and tried to bring it to their attention, they could be overloaded. This could also occur if a reader cast his 'profile' net too widely. If the cast is too narrowly defined, he might miss items of potential value to him.

Systems such as DICO and SASIDS could enable the community's network of channels to tune itself so that each member could 'define' the boundaries of his specialty so that he is neither overloaded nor missing many items he needs to know to keep up. But there is more to an integrated, viable scientific community than the ability of each member to cope by defining his specialty. The specialties somehow fit together, add up to a coherent process and legacy. Perhaps an analogy to Adam Smith's invisible hand ensures that, even though each scientist act, in his own interest to make discoveries, to specialize as much as he must, the result is the orderly 
growth of science. The secret lies probably in the way at least some scientists' minds are prepared to assimilate the discoveries reported by others (or their own) to form a richer, more comprehensive and coherent image. If an editor groups certain discoveries into coherent issues, he may stimulate this process. Or he may interfere with it, inhibiting it, by distracting certain readers away from more fruitful paths they might have followed.

Means for amplifying the way this process works well in successful scientific communities are needed if the analogy to a nervous system is to be justified. Unfortunately we do not understand how the process works. Research presently under way in our program is yielding clues about this discovery process. The reorganization or formation of new knowledge structures seems to be involved. This may require intense concentration of attention in individual minds on certain combinations of local or special knowledge structures. Several individuals more or less simultaneously focus on the same combinations by virtue of constraints on the search path that operate as filters on the literature, which, in turn, serve to channel and direct attention to the same area.

The leverage for amplifying this process may thus be in providing creative individuals with the opportunity to concentrate - to the exclusion of diversionary stimuli or a heavy load of distracting news and responsibilities. (The U.S.'s philanthropic MacArthur Foundation professes objectives very similar to sustaining such conditions). The literature system could provide such persons with ultra-sensitive filters that they can adjust to select only that which fits into their area of concentration for a period of time.

\section{From Nerves to Brain and Mind}

Could a scientific community evolve a communication-plus-knowledge processing system that is not only what a nervous system/brain is to a person but at a higher evolutionary level than that? Suppose that a future scientific community could count, among its members, agents with 'artificial intelligence' interacting with, perhaps supplementing, their human partners. An inquiring system of greater sophistication than Bacon (Simon, 1983, Science) could be built (Kochen, 1972). Computer-aided induction (Davis \& Lenat, 1982) might be able to accelerate or improve the community's production of discoveries.

In such a community, even as in today's information systems, the distinctions between communication and computing/knowledge-processing vanish. Communication, viewed as updating the preparedness of a recipient's mind for action or new knowledge, is an essential part of the process of increasing knowledge, understanding and wisdom. Minds of authors, readers, expert systems and communication networks will be integrated more fully. Out of this new structure would emerge new properties corresponding to all the higher mental functions now observed in individual persons: memory, attention, imagination, ability to anticipate the consequences of one's actions, to reflect con- sciously upon one's own mental processes.

Can functions now unavailable to the minds of individuals emerge as well? If so, what are they? It may be outside the range of my mental abilities to imagine it. But it seems worth the cost of setting up an experimental system in the hope and expectation of finding out.

The help of Charles Barr in preparing this manuscript is greatly appreciated, as is the support of a grant from the NSF, IST 8301505.

\section{References}

1. Davis, Randall and Lenat, Douglas. Knowledge-Based Systems in Artificial Intelligence. New York: McGraw-Hill, 1982.

2. Flood, M.M. and Kochen, M., Some Bibliographic and Sociological Devices to Improve Maintenance of Current Awareness About Literature. In: M. Kochen (Ed.), Some Problems in Information Science, New York: Scarecrow Press, 1965.

3. Garfield, E., Citation Analysis as a Tool in Journal Evaluation. Science 178: 471-479, 1972.

4. Kochen, Manfred, WISE: A World Information Synthesis and Encyclopedia. J. Docum., 28: $322-343,1972$

5. Kochen, M. and Tagliacozzo, R., Matching Authors and Readers of Scientific Papers. Infor. Stor, \& Retr., 10: 197-210, 1974.

6. Kochen, M., Technology and Communication in the future. J. of the Amer. Assoc. for Information Science, 32(2): 148-157, 1981.

7. Kochen, M. and Fredrickson, E., Interactions of Libraries and Publishers. Information Processing and Management, (6), 1981.

8. Kochen, M., Use of an Expert Nomination Network to Improve the Quality of Scientific Output. Address to Conference of International Science Editors, Philadelphia, MA 20, 1983.

9. Simon, Herbert, Bacon. Science, 1983. 